\title{
Wearables as Context for Guiard-abiding Bimanual Touch
}

\author{
Andrew M. Webb ${ }^{1,2}$, Michel Pahud ${ }^{1}$, Ken Hinckley ${ }^{1}$, and Bill Buxton ${ }^{1}$ \\ ${ }^{1}$ Microsoft Research, Redmond, WA, USA, \{kenh, mpahud, bibuxton\}@microsoft.com \\ ${ }^{2}$ Interface Ecology Lab, Department of Computer Science and Engineering, Texas A\&M University, \\ College Station, TX, USA, andrew@ecologylab.net
}

\begin{abstract}
We explore the contextual details afforded by wearable devices to support multi-user, direct-touch interaction on electronic whiteboards in a way that-unlike previous work - can be fully consistent with natural bimanualasymmetric interaction as set forth by Guiard.

Our work offers the following key observation. While Guiard's framework has been widely applied in HCI, for bimanual interfaces where each hand interacts via direct touch, subtle limitations of multi-touch technologies - as well as limitations in conception and design - mean that the resulting interfaces often cannot fully adhere to Guiard's principles even if they want to. The interactions are fundamentally ambiguous because the system does not know which hand, left or right, contributes each touch. But by integrating additional context from wearable devices, our system can identify which user is touching, as well as distinguish what hand they use to do so. This enables our prototypes to respect lateral preference- the assignment of natural roles to each hand as advocated by Guiard - in a way that has not been articulated before.
\end{abstract}

\section{Author Keywords}

Bimanual input; Guiard; direct touch; pen+touch; wearables

\section{ACM Classification Keywords}

H.5.2 [Information Interfaces and Presentation]: Input

\section{INTRODUCTION}

$\mathrm{HCI}$ is witnessing a proliferation of very large and very small form-factors in an ecosystem (or "society") of devices that complement one another. Whiteboards and tabletops offer multi-user interaction, while wearables such as watches $[3,17]$, fitness bands [22,50], and rings [58] promise personal interaction that is contextual in a fine-grained way.

In particular, during multi-user, multi-touch interaction on large displays, standard touchscreens rarely distinguish the contribution of individual users (much less the preferred vs. non-preferred hands) from one another. The problem is that all touches trigger the same events, generic "contacts" that

\footnotetext{
Permission to make digital or hard copies of all or part of this work for personal or classroom use is granted without fee provided that copies are not made or distributed for profit or commercial advantage and that copies bear this notice and the full citation on the first page. Copyrights for components of this work owned by others than the author(s) must be honored. Abstracting with credit is permitted. To copy otherwise, or republish, to post on servers or to redistribute to lists, requires prior specific permission and/or a fee. Request permissions from Permissions@acm.org.

UIST 2016, October 16 - 19, 2016, Tokyo, Japan

Copyright is held by the owner/author(s). Publication rights licensed to ACM. ACM 978-1-4503-4189-9/16/10 ..\$15.00

DOI: http://dx.doi.org/10.1145/2984511.2984564
}

seem identical without further context [19,39,47]—namely, who touched the display, and which hand produced the event. This missing context is especially problematic for bimanual interaction on large displays, as such a setting compounds these ambiguities. And while wearables are just one possible approach to sense such context, they do offer an expedient solution that rides on existing trends towards a society of devices where users carry, wear, or encounter many interconnected devices - a mobile approach that scales to situated displays (rather than requiring new sensors at each location).

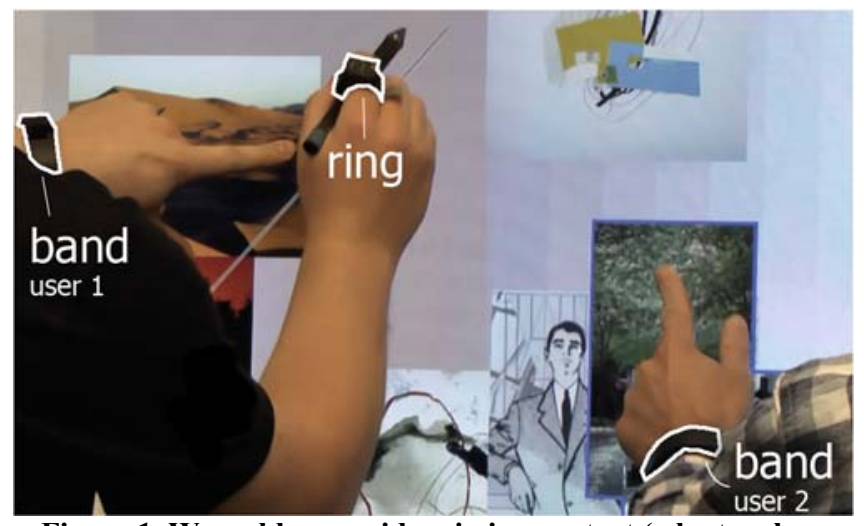

Figure 1. Wearables provide missing context (who touches, and with what hand) for direct-touch bimanual interactions.

We demonstrate that augmenting touch with such context supports phrasing together [13] inputs in a manner consistent with bimanual asymmetry as set forth by Guiard [27]. Our key point is that this added context-who touches and with which hand - enables design of interactive dialogs that can flexibly assign appropriate roles to each hand, including symmetric role assignments where appropriate. Because of the arbitrary limitations and ambiguities inherent in standard multi-touch technology, most previous systems exploring bimanual touch - including efforts motivated by Guiard's insights - have not been able to fully support this design goal.

In the remaining sections of this paper, we support our central contribution:

...the use of wearables to identify which user, and distinguish what hand, is touching a large display —and to thereby phrase together direct-touch interactions in a manner consistent with the bimanual-asymmetric assignment of roles to the hands-right vs. left, preferred vs. non-preferred — as set forth by Guiard...

...as follows: First, we unpack in greater depth Guiard's bimanual-asymmetric class of interactions [27] and we 
connect this with phrasing [13]. Second, we review related work. Third, we detail the design of Affinity, which uses a single wearable in combination with phrasing to support bimanual-asymmetric interactions. We describe a few details of how we use wearables to identify users and sense hands (details largely explored already by others, and which are therefore not our key contribution). Fourth, we discuss MoodBoard, which uses two wearables to explore further possibilities. And fifth, we offer an informal evaluation and discuss some implications of our work.

\section{BACKGROUND THEORY AND MOTIVATION}

Our research is founded on key insights regarding human bimanual action articulated by Guiard [27], as well as the interaction design strategy of chunking and phrasing [13].

Guiard's Theoretical Framework of Bimanual Asymmetry Guiard investigates human bimanual specialization. He emphasizes the logic of the division of labor between the hands, a view that has widely influenced the design of twohanded interfaces. Of especial interest to our work, Guiard introduces lateral preference, as well the principles of LeftHand Precedence and Right-to-Left Spatial Reference.

\section{Lateral Preference}

As opposed to the study of 'manual superiority,' which (perhaps misguidedly) frames one hand as 'superior' to the other, Guiard favors lateral preference, which frames twohanded action in terms of complementary roles for the hands:

in a task consisting of two differentiated manual roles, $A$ and $B$, there are two possible ways to assign role $A$ and role $B$ to the left and right hands (A-left and B-right, or $A-$ right and $B$-left).

For standard multi-touch input, even if the designer desires specific roles (A vs. B) for the hands (right vs. left, preferred vs. non-preferred), this desire is oft-undermined by the inability to determine which hand touches the display. Furthermore, if the user is unknown, the mapping of right= preferred and left=non-preferred (or vice-versa) injects further ambiguity during multi-user input. Thus, standard multi-touch cannot sense or abide by any particular choice for $A$-left and B-right versus $A$-right and $B$-left. The role of the hands (lateral preference) is fundamentally ambiguous.

\section{Left-Hand Precedence}

Guiard observes that (for right-handers) the left hand tends to lead the right in its contribution to a joint activity. For example, the left hand positions and orients a sheet of paper, then the right hand begins to write. In general, Guiard codifies this as the Left-Hand Precedence principle.

But users may touch with the preferred hand in isolation. If the touch cannot be distinguished as left vs. right, it remains ambiguous from the system's viewpoint. Is this indeed an isolated touch of the preferred hand? Or is it perhaps a nonpreferred hand touch, which via Left-Hand Precedence should be interpreted as preparatory to the (complementary) action of the preferred hand? The system has no way of discerning the user's intent at the onset of the first touch.
For example, Kharrufa et al. [39] identify the supervisor in a classroom setting by instrumenting the supervisor's preferred hand with a wearable. A regular touch might be the supervisor's non-preferred hand, or it might be another (uninstrumented) user. This therefore fails to enable bimanual interactions that lead with the non-preferred hand; the Left-Hand Precedence principle cannot be satisfied.

\section{Right-to-Left Spatial Reference}

The principle of Right-to-Left Spatial Reference states that "motion of the right hand typically finds its spatial references in the results of the motion of the left." This means that the left hand generally should establish (and frame) the interaction, relative to which the right hand takes action.

Duet [17], for example, goes beyond [39] by augmenting the left hand with a watch, and then using this wearable to sense when the non-preferred hand touches a screen. Which sounds great. However, this is still not satisfactory because Duet realizes this capability in the context of a pair of devices, the watch plus a mobile phone, and the phone therefore has to be held in the right, preferred hand while the left, non-preferred hand refers to the contents on the display. In other words, the left refers to the right, which is exactly the reverse of the natural roles specified by Right-to-Left Spatial Reference. Thus, Duet (unlike our work) cannot support bimanual interactions that are consistent with the principles of asymmetric manual activity as set forth by Guiard.

\section{Symmetric vs. Asymmetric Bimanual Interaction}

While Guiard focuses on asymmetry, he does note that certain acts, such as lifting weights or turning a steering wheel, often invoke symmetric action of the hands. And certain idiomatic multi-touch gestures on large displays, such as bimanual shrink and stretch, show that symmetric mappings have utility as well. However, by their very nature symmetric mappings employ an interchangeable assignment of roles to the hands. This can mask a touchscreen's underlying inability to differentiate left from right. DiamondTouch [19], for example, can sense when different users touch, but cannot distinguish which hand (left or right). This doesn't matter for symmetric mappings, but ambiguities still lurk: the designer cannot realize differentiated touches that fully abide by both Left-Hand Precedence and Right-toLeft Spatial Reference - nor support a flexible interleaving of symmetric and asymmetric interactions, as our work does.

Phrasing Activity of the Right Hand together with the Left Phrasing uses muscular tension (which may be nothing more than a light touch to maintain contact with a touchscreen) as a way to tie together a series of inputs into a single, cohesive gestural phrase [13]. Building on top of Guiard, then, during bimanual interaction the left hand establishes and "holds down" the phrase. The right hand then inserts its actionswhich may consist of multiple taps, drags, or other gestures - into the reference frame specified by the left hand, and which thereby collectively gives those actions a specific interpretation, or meaning. 


\section{Using Theory to Inform \& Motivate Our Techniques}

Our analysis highlights a novel design direction that uses identification of users, and differentiation of the hands, to phrase together bimanual interactions in a manner consistent with Guiard. Previous multi-touch systems have lacked this context, or have not fully considered its implications for the design of bimanual interactions. By surfacing these ambiguities - and showing how subtle some of the issues are in terms of what lateral preferences can be supported-our work opens up new possibilities and design insights for direct-touch bimanual interaction on large displays.

\section{ADDITIONAL RELATED WORK}

Our work builds on insights from bimanual input, wearables (and other sensors) plus touch, and large-display interaction.

\section{Asymmetric (and Symmetric) Bimanual Interaction}

Guiard's work has motivated many techniques $[30,33,40]$. Yet the difficulty inherent in unambiguously supporting proper lateral preferences for standard direct-touch input has not (to our knowledge) been previously articulated.

Direct vs. Indirect, Mechanical Intermediaries, Pen vs. Touch It is sometimes possible to design around hand ambiguity by favoring symmetric bimanual interactions [34,40,43,64], with interchangeable roles of the hands. But, it is telling that many previous bimanual-asymmetric techniques do not use direct touch but instead rely on mechanical intermediaries such as pens [11,33] (or indirect input devices including trackballs [9], or pucks [40,43], or touchpads [15]) that distinguish the contribution of the left hand from that of the right. However, even predominately asymmetric designs such as Toolglass often interleave symmetric interactions [9,37], so flexibly mixing both styles (symmetric and asymmetric) is important to support as well.

Pen + touch together $[11,30,33,49]$ is an interesting test case. Here, direct-touch is present, but typically only strokes articulated by the pen-and not touches with the pen temporarily "tucked" in hand - can definitively be associated with the preferred hand [32]. Conté [60] offers a rich example of this style of interaction, but it must initiate many of its interactions via the digital Conté crayon in the preferred hand to do so. Thus, many pen+touch systems still face ambiguities in terms of Left-Hand Precedence and Right-toLeft Spatial Reference for touch, and in general it has not been recognized that the inability to distinguish left vs. right limits the interaction fidelity vis-à-vis Guiard.

\section{Symmetric Interaction}

Through interchangeable roles of the hands, symmetric bimanual interactions afford parallel action. This can take the form of two independent movements, such as dragging a pair of objects to two different locations [16], or the movements may comprise elements of a compound task, as in twohanded stretchies [9,37], or spline curve manipulation [42]. Task difficulty, divided attention, and visual integration can all influence the degree of parallelism attainable [6]. By distinguishing the hands, our work demonstrates how both asymmetric and symmetric interactions-potentially with either strict or flexible assignment of roles to the hands - can be richly supported through appropriate context sensing.

\section{Wearables (and Other Sensors) Plus Touch}

Although our insights are not particularly wedded to wearable devices, a number of previous systems do leverage personally-worn devices to augment touch and multi-touch interactions. Wrist-worn devices on either the preferred or non-preferred hand (but not both) have been used in combination with touch on phones [17] and to authenticate users $[39,48]$.

Vision-based techniques $[20,55,66]$ or proximity sensors [2] can sense which hand approaches a touchscreen. However, it remains difficult to bridge the "identity gap" of associating tracked objects (blobs) with a specific individual. Biometric properties inherent in touch [10], capacitive coupling [19], and combining inertial sensors on mobile devices with vision-based approaches [57] offer other possibilities to associate touches with an individual. Fingerprint sensing [35] or fiduciary-tagged gloves [47] can distinguish the hands and even individual fingers, as can instrumenting fingertips with optical sensors [65], but these efforts do not unpack what this means for bimanual interaction in depth.

Identifying, articulating, and exploring the implications of this added context (rather than the particular manner in which it is sensed) is the focus of our contribution.

\section{Interaction on Large Displays}

Large displays afford workspaces for collaboration and creative work [4]. Their scale affords use of both hands by multiple users, but previous work has not remarked upon the inherent ambiguities in multi-touch with respect to bimanual input. For large public displays, breakdowns in personal space may occur, where users will reach across each other to interact with objects, causing sensing and interaction design issues [5]. Affinity primarily relies on phrasing [13] to avoid this, where personal spaces are delineated by activating a tool sheet with the non-preferred hand (Figure 2); the system assumes users do not reach into one another's tool sheets. In MoodBoard, we avoid the issue completely by leveraging two wearables, one on each hand, to disambiguate touches.

\section{Electronic Whiteboards}

Interactive wall-sized displays can bridge the gap between informal whiteboard use and the rigid data manipulations of the desktop [28]. Tivoli extends the functionality of physical whiteboards with digital affordances, while preserving an informal feel [54]. Flatland provides functionality for managing space, with flexibility for different applications [52]. Range uses implicit interactions to transition the whiteboard among different modes based on proximity [36]. Our work, by contrast, focuses on the role of bimanual directtouch (and pen) interaction for design activities.

\section{Design and Mood Boards}

We explore our techniques in the context of mood boardsassemblages of images, sketches, and other visual media that designers use in studio environments for both problem- 
finding and problem-solving [24,45] by drawing connections among ideas through visual juxtaposition [12]. Portfolio Wall, for example, enables designers to drag images from personal computers to a wall-mounted display [14]. Funky Wall [46] and MessyBoard [21] offer other examples, but our exploration of Affinity and MoodBoard offers the only example that leverages identified touches for bimanual input.

\section{Tabletops}

Although our focus is on vertical displays, and electronic whiteboards in particular, our work is heavily informed by related techniques drawn from tabletops. For example, bimanual interaction has been extensively explored in this context, including whole hand gestures [64] and cooperative gestures [51]. Some systems can differentiate the hands, for example using different parts of the hands to perform different operations [47], or moving individual elements with the preferred hand and groups of elements with the nonpreferred hand [2]. However, these previous systems do not address the implications for bimanual touch as we do.

\section{Implications}

As should now be clear, despite many previous efforts that have addressed bimanual interaction, direct touch, and touch augmented with various forms of sensing, to our knowledge no previous system has put all of these elements in play simultaneously. In particular, our work is the first to articulate the underlying ambiguities that direct-touch presents with respect to lateral preference, i.e., the proper assignment of distinct roles to the hands during asymmetric bimanual interaction. Without a clear understanding and enumeration of these issues, it is difficult to design systems that adhere to the principles of asymmetry as articulated by Guiard - much less to contemplate designs that explore other mappings, or that (intentionally) diverge from the Guiardabiding perspective in service of other design goals.

In the following sections, our techniques demonstrate various design considerations and trade-offs for such interactions, including the interleaving of bimanualsymmetric inputs. And to be absolutely clear, the contributions of the techniques are in their illustration, exploration, and bracketing of various design decisions in these regards, rather than in the underlying gestures themselves (taps, drags, chops, and so forth). Collectively, these interactions show in some detail why it is problematic to design bimanual interactions that incorporate Guiard's well-known principles without sufficient information on which user and which hand-information that is sorely lacking on almost all touch displays in common use.

\section{AFFINITY: INSTRUMENTING LEFT HAND}

Our investigation began with designing a user experience for bimanual-asymmetric interaction involving one wearable per user. We developed Affinity, a prototype environment for a pair of users to author affinity diagrams [8], where the left (non-preferred) hand of each user is instrumented with a wearable sensor, the Microsoft Band [50]. Affinity diagrams involve recording ideas on post-it notes, reflecting on the relationship among ideas, organizing ideas into groups, and drawing lines and shapes to convey connections [8].

\section{Bimanual-Asymmetric Interaction}

In Affinity, a user creates digital post-it notes and shapes with bimanual-asymmetric gestures through an in-place tool sheet. The tool sheet is activated with a touch from their left hand (Figure 2, top-left), following Guiard's principle of Left-Hand Precedence. The wearable identifies the specific user that touched, presenting that user's name above the tool sheet and a personalized set of commands (Figure 2, bottom). The tool sheet can be moved by dragging the left hand touch. The user selects a colored note or shape command in the tool sheet with their right (preferred) hand, following Guiard's principle of Right-to-Left Spatial Reference. A new note or shape appears next to the tool sheet.

\section{Combining Asymmetric and Symmetric Interactions}

Command selection is asymmetric, where the left hand activates a tool sheet and the right hand selects a command, such as Draw Rectangle. In Affinity, once a command is selected, the two hands function symmetrically to manipulate parameters of the command in parallel, such as scaling and positioning rectangles [43], lines, or circles.

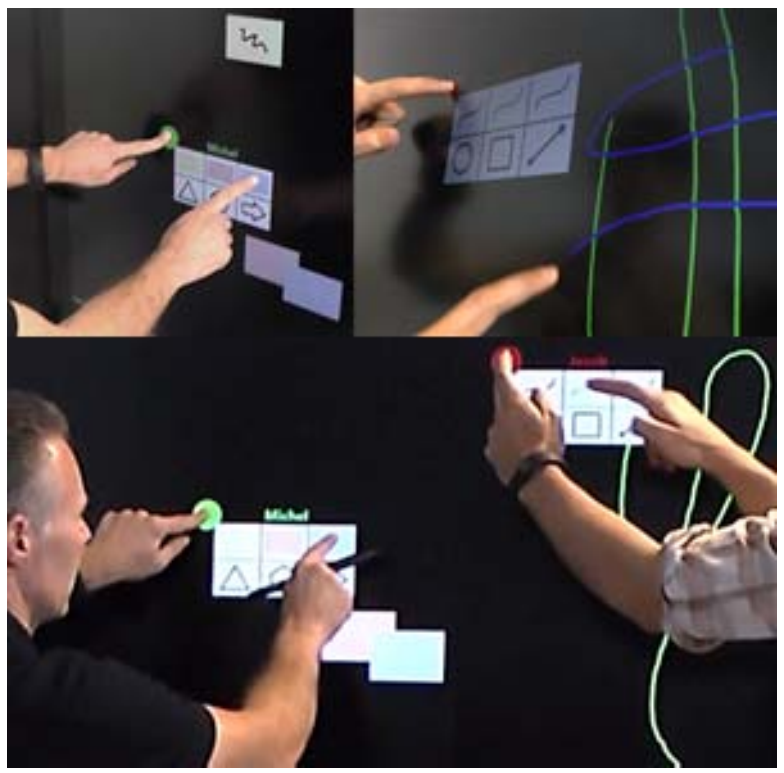

Figure 2. Snapshots of Affinity. Touches with the left hand identify the user and present that user's unique tool sheet, while touches with the right hand select tools, draw, and move notes. User's name and color are shown above each tool sheet.

\section{Unimanual Interaction: Right Hand}

Notes and shapes can be freely arranged to organize ideas by dragging them (via touch) with the right hand. The pen is used to draw or write ideas inside of notes. The user can also draw colored strokes to connect notes using the pen or a touch on an empty area of the space with their right hand.

Wearable + Phrasing $=$ Guiard-abiding Bimanual Touch In Affinity, we employ only one wearable. This level of sensing is sufficient to identify and differentiate any interactions initiated by the left hand. And the design of the 
interactions is such that the left hand leads all bimanualasymmetric transactions (Left-Hand Precedence).

Although the right hand remains uninstrumented, Affinity's design limits the potential of this to cause problems. Given users' reluctance to invade the personal space of one another [26], once the left hand establishes a frame of reference (in the form of the in-place tool sheet), it is reasonable to assume that an uninstrumented touch in the tool sheet is most likely the right hand of the same user. Thus, the role of the right hand can be distinguished (within reasonable assumptions) for such transactions even though it is not directly sensed.

Note that this also leverages Buxton's notion of phrasing to connect the activity of the right hand to the reference frame established by the left hand. As long as the left hand remains in contact with the display, the "phrase" remains open, allowing the right hand to continue the transaction (i.e. by dragging "through" the tool sheet called up by the left hand.)

As noted above the design also supports unimanual actions by the right, uninstrumented hand, such as moving around the virtual post-it notes on the display. The system knows that an uninstrumented touch is not performed by the left hand, and therefore it can unambiguously interpret the input using the unimanual, preferred-hand role. Touching with the left hand would trigger a bimanual-asymmetric transaction, i.e. bring up the tool sheet, instead.

Of course, inputs articulated by any walk-up users who are completely uninstrumented would remain ambiguous. However, the fallback of the system here-namely, to interpret all such transactions as unimanual inputs - offers a reasonable fallback, and indeed there would seem to be some virtue in only allowing authenticated users to access the full functionality of the application as manifest in the tool sheets.

Thus, with careful attention to detail in the design, even a single wearable can support a significant range of interactions that adhere to Guiard's principles.

\section{Hardware and Signal Processing}

Affinity consists of the following hardware components: (1) a large 55-inch pen and multi-touch display on a vertical stand; (2) two Microsoft Bands; and (3) a computer with Bluetooth for processing band sensing data and running our affinity diagram application.

The Microsoft Band is a fitness band worn around the wrist. Its sensors include a 3-axis accelerometer and gyroscope. The Band communicates wirelessly with other devices via Bluetooth, and has a sampling rate of $60 \mathrm{~Hz}$.

We use accelerometer signals from the band to detect the hard-contact forces (spikes) that result from the hand coming into contact with the display. We identify touches with the left hand by detecting spikes of a particular size and width in the band's accelerometer signal and associate those spikes with touch-down events. These spikes are larger and quicker (narrower pulse width) than normal hand movements, which exhibit more gradual changes in acceleration.
We look at the $\mathrm{x}$-axis of the accelerometer, which is the axis parallel to the orientation of the forearm when the device is worn. Similar to the spike detection in $[32,44]$, we detect spikes by sampling for a bump (above a threshold) in the second order finite difference with a window of $100 \mathrm{~ms}$ (Figure 3). If a spike is within the $100 \mathrm{~ms}$ window following a touch-down event, we associate that touch to the left hand. If a touch-down event occurs, but there is no spike within $100 \mathrm{~ms}$, we associate that touch to the right hand.

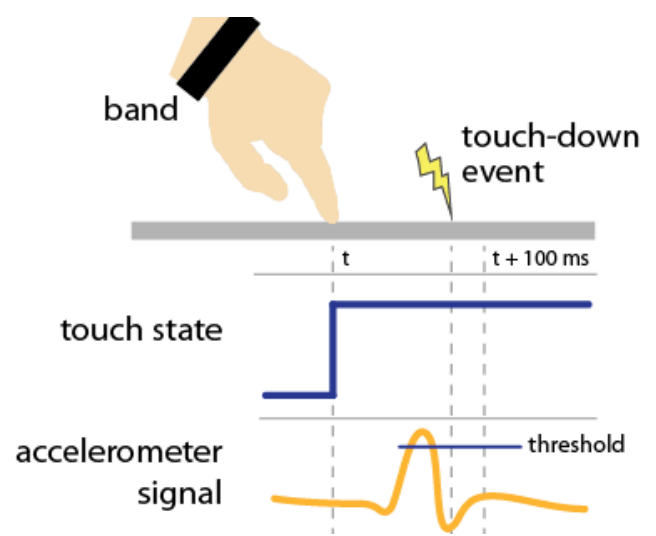

Figure 3. Diagram of signal processing for identifying touches based upon spikes in accelerometer signal.

\section{MOODBOARD: INSTRUMENTING BOTH HANDS}

We further demonstrate how wearables - this time one on each hand-support Guiard-abiding bimanual-asymmetric interactions on large displays. We present MoodBoard, a prototype environment for authoring mood boards with a single user or pair of users.

As with Affinity, we instrument the left (non-preferred) hand with a Microsoft Band. We instrument the right (preferred) hand with a different wearable, an in-house designed ring, placed on the index finger. We intentionally use two types of wearables. In everyday life, it seems unlikely that a user would choose to wear two watches, or two fitness bands, but it may be more plausible that a user would own two different (and complimentary) wearables, such as a fitness band on the non-preferred hand and a "smart ring" type of device $[38,53]$ on the index finger of the preferred hand.

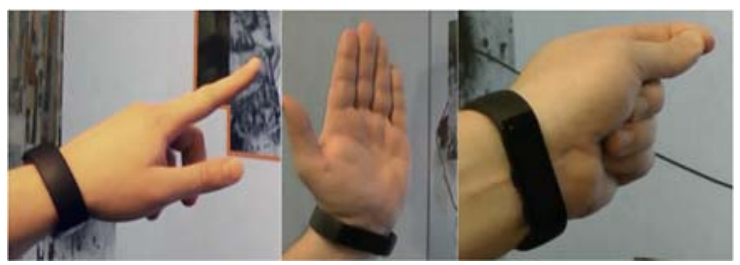

(a) (c)

Figure 4: Detected orientations: (a) normal, (b) side, (c) back.

In MoodBoard, we use the orientation of devices as an additional parameter for interaction [63]. We detect three orientation states: normal, side, and back (Figure 4). Normal orientation is the standard touch orientation when touching with the tips of fingers. Side orientation is when the pinky finger side of the hand is used. Back orientation is when the 
back of a finger is pressed against the display. Details of our orientation detection methodology are presented later.

We designed a suite of interaction techniques for creating and organizing content in a mood board. The techniques demonstrate various considerations and trade-offs for Guiard-abiding bimanual touch interaction on large displays. Our core interactions are well-known gestures drawn from prior work; it is the way we augment them with the added context of which user and which hand - and how we use this to illustrate various design choices - that is new. These techniques further demonstrate how additional context from wearables, including orientation of the hand-in conjunction with Guiard's principles - can support phrasing together rich, bimanual-asymmetric interactions. Adding a wearable on the right hand addresses ambiguity issues for lateral preference, removing the need for any assumptions about what an uninstrumented hand represents, and thereby affording a richer design space that can assign a variety of distinct, complementary roles to each hand.

In the sections that follow, we present a series of interactions that we prototyped within MoodBoard, with the intent of exploring this design space and the various possibilities that it offers designers. Our intent is not to argue that any one particular technique represents the epitome of our approach, but rather to cover enough of the design space to compare and contrast various design issues, as well as to illustrate that many novel bimanual-asymmetric possibilities arise.

\section{Chopz}

Elements in a mood board can overlap. The user needs techniques for adjusting the visual stacking order (or zindex), so that the desired elements can appear on top of or below other elements.

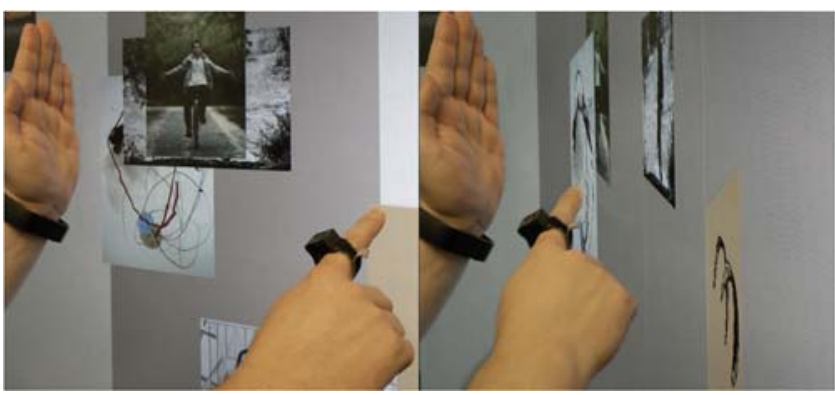

Figure 5. Example of ChopZ. Left: Using right hand to select scope after chop with left hand. Right: Using right hand to move individual elements to change visual stacking order.

Design tools, such as Adobe Illustrator [1], use an ordered list to represent visual stacking order. In the list, elements are organized top to bottom where elements higher up in the list appear above elements lower in the list. Visual stacking order is changed by reordering the list using drag and drop. The list appears in a floating palette near the display edge. With large displays, with multiple users, a floating palette near the display edge is not easily accessible.
Contextual menus, such as those in Microsoft PowerPoint, can allow adjusting visual stacking order of an element in discrete steps: bringing it up one step, down one step, all the way to the top, or all the way to the bottom. Getting the desired stacking order with this approach can be tedious as the discrete changes may require multiple executions.

In-place techniques allow adjusting visual stacking order with mouse or pen input [56]. Touch pressure has been used to adjust visual stacking order between two elements [18].

ChopZ is a bimanual-asymmetric interaction technique for adjusting the visual stacking order of elements. It allows for reordering of elements using drag and drop, while supporting in-place activation and selection of elements. ChopZ enables multiple users to perform the operation simultaneously.

ChopZ is activated with a side-of-hand "chopping" gesture $[49,64]$ using the left hand (Figure 5). The chop gesture phrases the interaction. While the chop is held down, any right hand actions are input for ChopZ. Releasing the chop, immediately deactivates ChopZ and ends the interaction. This follows Guiard's principles of Left-Hand Precedence and Right-to-Left Spatial Reference. The left hand begins the interaction with a chop gesture. The right hand defines the bounds of selection in reference to the left hand chop.

After chopping, the user touches down with their right hand to define a scope of elements on which to interact. The selection area is visually represented by a gray rectangle, vertically bounded by the top and bottom of the display and horizontally bounded by the two hands (Figure 5, left). Moving either hand adjusts the horizontal bounds of the selection area. The user may perform a bimanual-symmetric gesture by moving both hands together. A flick gesture with the right hand away from the left hand will automatically select all elements in the direction of the flick. We suspect that flick selection will be particularly useful on large displays where the reach of a user is smaller than the display size. Lifting up the right hand touch, finalizes the selection.

Upon finalizing selection, an animated transition (500 ms) occurs, rendering a view of the selected elements as if seen from the side of the board. In other words, the x-axis is remapped to the $\mathrm{z}$-axis, and a perspective transformation is applied to the elements to convey a side view (Figure 5, right). As a result of the re-mapping, elements are ordered in the $\mathrm{x}$-dimension according to their visual stacking order. Elements at the top of the stack appear on the left-most side, and elements at the bottom appear on the right-most side.

With the right hand, users can drag elements to adjust their visual stacking order. Moving elements to the left, moves them on top of elements to their right.

After the user is finished adjusting elements stacking order, they can lift up their left hand, deactivating ChopZ. An animated transition $(500 \mathrm{~ms})$ returns the selected elements to their original positions, although with a new stacking order based upon the user's changes. 


\section{Straightedge Tool}

A straightedge is a valuable tool in visual design, enabling designers to draw straight lines that create visual structure, such as showing connections among elements or producing geometric shapes on which to align elements around. Straightedges also enable aligning elements along a line.

We present a bimanual-asymmetric interaction technique for using a straightedge that follows Guiard's Right-to-Left Spatial Reference principle. The left hand gestures to create a movable virtual straightedge. Then, using the right hand, a user can draw straight lines using a pen, or move elements parallel to the straightedge using touch.

The straightedge tool is activated using a two touch gesture with the index finger and thumb of the left hand (Figure 6). This gesture phrases the straightedge operation for the user. While maintaining this gesture with the left hand, any touches or pen input with the right hand by this user will parameters for the straightedge operation.

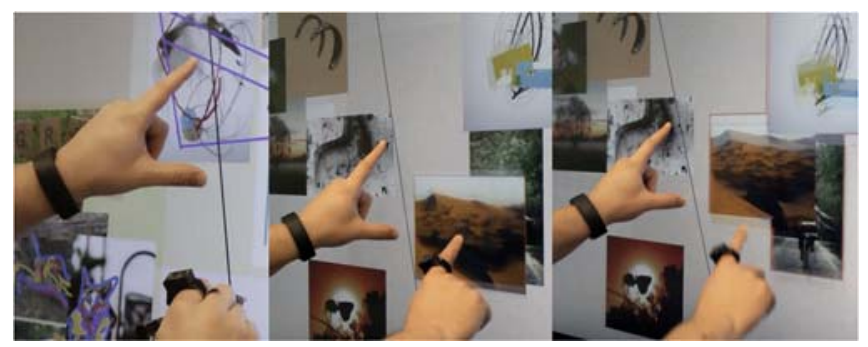

(a)

(b)

(c)

Figure 6. Straightedge tool activated with left hand. Input modality with right hand and context specify operations: (a) using pen draws a straight line; (b) using touch on an image moves that image along the line; (c) using touch on the background moves the entire space on that side of the line.

Upon activation, a thin line representing the straightedge appears near the left hand. Moving the left hand translates and orients the straightedge line, just as if holding a physical straightedge tool. The line extends from one edge of the display to another. The slope of the line is defined by the two touch points. We offset the straightedge line by 40 pixels to avoid occlusion with the hand. The direction of the offset is determined by the orientation of the band to ensure that the line appears away from the hand instead of under it. This shows how implicit parameters of the Band enrich the interaction. When gravitational forces on the y-axis of the accelerometer read greater than 0.5 or less than -0.5 , we position the line to the left of the hand; otherwise, we position the line to the right.

With the straightedge activated, the user can draw ink with the pen (in the right hand). The ink is snapped to the straightedge line, allowing for the creation of straight lines (Figure 6a). Alternatively, the user can use touch with the right hand to select a single element and move that element along a line parallel to the virtual straightedge (Figure 6b). If the user touches on an empty area of the mood board, they can move all elements on that side of the line parallel with the straightedge (Figure 6c). This enables quickly shifting large regions of elements around on the mood board to make space for new content without having to make a selection.

\section{Group Sheets}

As connections emerge while mood boarding, a user may wish to group together a set of elements that may or may not overlap so that those elements are explicitly connected, allowing the user to move those elements together without losing their relative positioning with each other.

Group sheets are a pen+touch interaction technique for defining persistent groups of elements. A group sheet represents a group of elements as a rectangular, translucent region (Figure 7).

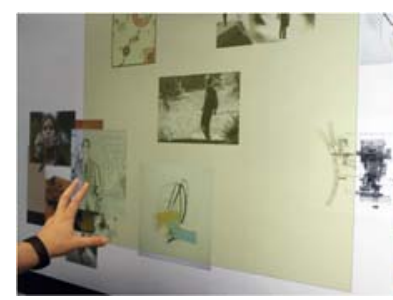

(a)

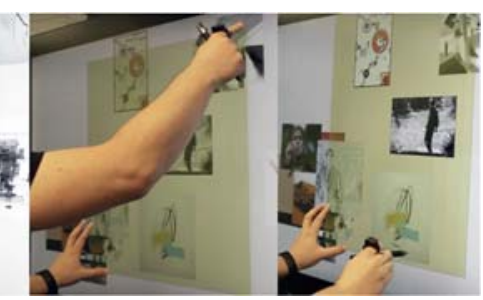

(b)

(c)

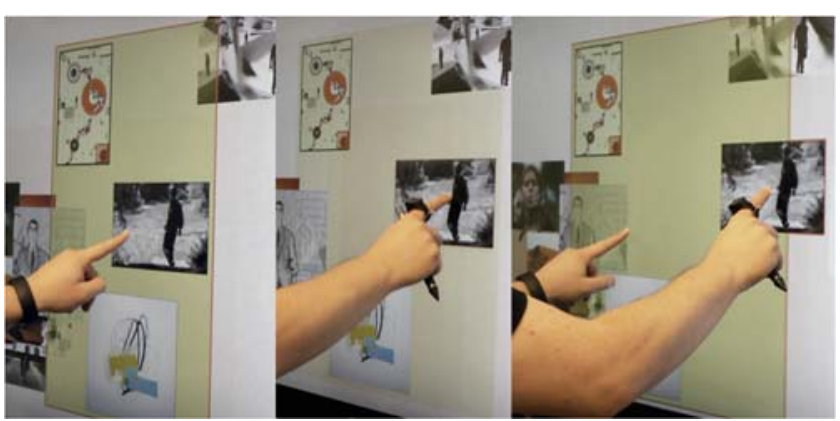

(d)

(f) (e)
Figure 7. States of group sheet creation (a-c) and editing (d-f): (a) three finger right angle gesture with left hand creates a new sheet; (b) bimanual-symmetric gesture adjusts size and position; (c) trace over elements with pen to add to sheet; (d)

move entire sheet with left hand; (e) move an individual element with right hand; (f) edit group contents by phrasing with left hand and dragging an element out with right hand.

\section{Phrasing: Sheet Position, Size and Contents}

Creating a group sheet begins with a three-finger right-angle gesture with the left hand (Figure 7a). A sheet with a unique background color (with $43 \%$ opacity) appears. Position of the right angle gesture defines the bottom-left corner of the sheet. This left hand gesture phrases Group Sheet transactions. While the left hand remains down, the user can modify the sheet's position and size using both hands through bimanual-symmetric interaction, and add elements to the sheet using the pen in the right hand.

Using a touch with the right hand, the user can specify the opposing top corner of the sheet (Figure 7b). Moving both hands simultaneously defines the position and size of the sheet, based upon the positions of the left and right hands. 
With the right hand, the user marks over with a pen (Figure 7c) or taps with touch elements to add them to the sheet. A short mark or tap is enough to add an element quickly. However, for selecting thin ink strokes that are difficult to precisely mark with pen or touch, the user can also trace over the ink stroke with the pen. This ensures that the stroke is selected, and not elements behind the stroke that were accidentally traced over. On pen up, whatever elements the user marked are added to the sheet. Lifting up the left hand ends group sheet creation.

Touching the sheet again with the left hand selects the sheet and activates an edit mode. The user can change which elements are in the sheet using the right hand. With the pen, the user can mark over elements to toggle adding or removing them from the sheet. With touch, elements can be removed by directly dragging the element out of the sheet (Figure 7f), or conversely, holding the element in place while dragging away the sheet with the left hand.

\section{Unimanual Interaction: Positioning}

We use different hand mappings for spatial positioning of sheets and elements (Figure 7d-e). With the left hand, the user positions an entire group sheet, and with the right hand, the user positions individual images within a group sheet. These mappings are consistent with our other unimanual interactions beyond group sheets (see Unimanual section).

\section{Tool Sheets Revisited: Ink Styling}

In Affinity, left hand touches activate an in-place tool sheet. A single touch provided an easy way to activate tool sheets, but in MoodBoard, a single touch is used to move elements around. Positioning elements is a critical operation for mood boards to support juxtaposing ideas. However, we can take advantage of our ability to sense orientation of wearable devices to still use a single touch, but with the back of the finger, to activate the tool sheet.

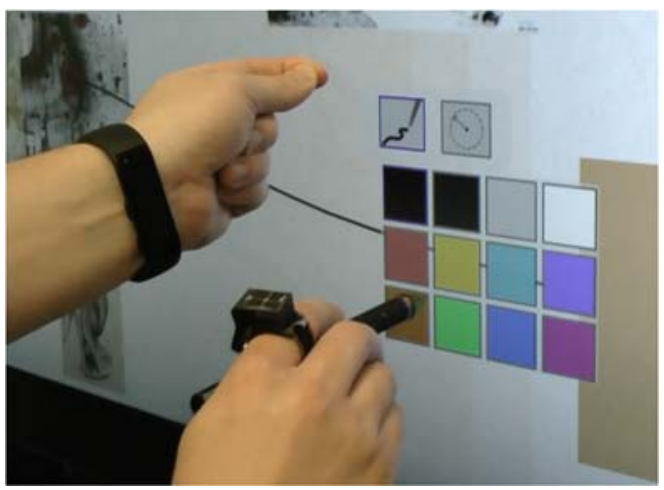

Figure 8. Tool sheet activated with back of finger gesture using left hand. User can select color and adjust brush size.

An in-place tool sheet is activated with the left hand by pinching together the index finger and thumb and pressing the back of the index finger at the fingernail against the display (Figure 8). We designed this interaction with a physical mapping, where pinching the index finger and thumb simulates holding the top corner of the tool sheet, as if it were a physical sheet. Dragging the activating touch, moves the tool sheet, maintaining the relative positioning between touch and tool sheet. Lifting up the activating touch deactivates the tool sheet, removing it from the display.

The mood board's in-place tool sheet enables changing the color and size of ink strokes (Figure 8). The tool sheet is interacted with using pen or touch with the right hand. The user can draw ink strokes while the tool sheet is active, allowing the user to quickly create multi-colored drawings without having to repeatedly activate the tool sheet.

\section{Unimanual: Spatial Positioning}

MoodBoard also supports unimanual interactions. Since we can identify the hands, we can map different actions to each hand. We map spatial positioning of individual elements to the right hand, while we map positioning of groups to the left hand. Others have used a similar mapping [2].

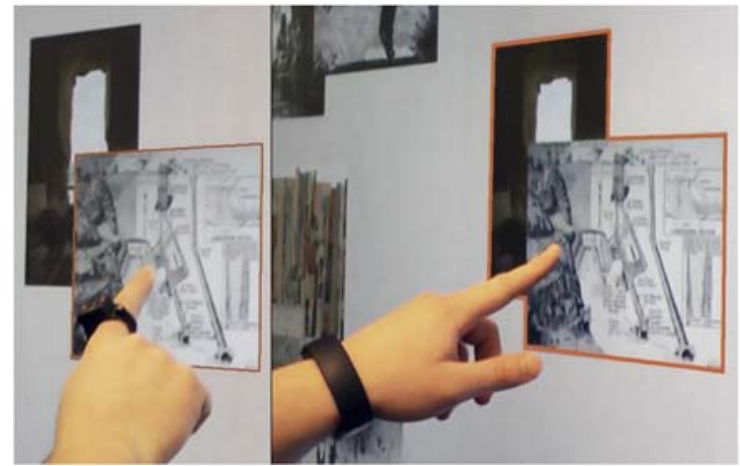

Figure 9. Unimanual positioning. Left: Right hand selects single element on top (thin orange outline). Right: Left hand selects two overlapping elements (thick orange outline).

Arranging the spatial positioning of elements within a mood board is one of the most basic and frequently performed actions. In MoodBoard, individual elements are freely positioned using a single finger touch and drag with the right hand (Figure 9, left).

Overlapping elements visually form groupings through their spatial contiguousness, similar to a roughly arranged pile of photos or documents. The lack of white space between these elements connects them as a whole. A single touch and drag on an element with the left hand moves the touched element and all elements which it overlaps or are overlapped by it while maintaining their relative positions (Figure 9, right).

We use visual feedback to differentiate individual element selection from overlapping group selection. For single elements, a thin one-pixel line outlines the selected element. For overlapping groups, a thicker four-pixel line contours the contiguous group of elements. The color of the feedback matches the color assigned to a user.

Bimanual Interaction through Phrasing Unimanual Together We address lateral preference by assigning distinct roles to the hands for spatial positioning. The fine-grain positioning of individual elements is assigned to the right hand. While, the coarse group positioning is assigned to the left hand. 
These role assignments can be combined to support bimanual-asymmetric interactions without need for any additional gesture or phrasing. For example, in some cases, an element may overlap with others, but the user does not wish to move that element with the others. The two hands may work together. The left selects an overlapping group, and the right hand pulls an element away from the group (with a drag), removes an element from the selection (with a tap) or holds an element in place (with a press and hold) while the left hand drags the group away.

\section{Hardware and Signal Processing}

MoodBoard consists of the same hardware components from Affinity with the addition of the ring. The ring contains an inertial measurement unit (IMU), wireless transmitter for sending data from the IMU, and a Velcro strap for fastening it to the user's finger (while easily accommodating variable sizes). The ring samples the three-axis accelerometer, gyro, and magnetometer embedded in the IMU at $90 \mathrm{~Hz}$.

We build upon our signal processing techniques from Affinity. Again, we identify which user is touching and with what hand by detecting spikes in the accelerometer signals from the wearable devices. Signals from both wearables are passed through a high-pass filter to remove effects of slow changing forces, such as gravity. The $\alpha$-values for our high pass filter are 0.75 for the band and 0.9 for the ring.

We can identify orientation by looking at the gravitational force on a wearable device. We apply a low-pass filter to the accelerometer signal to isolate slow changing, gravitational forces. The $\alpha$-value for the low-pass filter is 0.1 for both wearables. We define a vector for each orientation that describes that orientation in terms of gravitational force. We subtract the current gravitational vector (result of low pass filter) from each of the orientations' vectors, and observe the smallest difference. The orientation with the smallest difference is defined as the current orientation.

\section{PRELIMINARY USER EVALUATION}

We ran a preliminary informal study with 8 participants (4 female, all right-handed, 26-66 years old). All participants had experience using touch devices, but minimal to no experience using large displays. The goal of the study was to gather user feedback on our interaction techniques. Before investigating the more challenging multi-user context, we first wanted to better understand individual user experience. Thus, study sessions involved only one participant.

Participants were given an introduction to MoodBoard and a step-by-step explanation and demonstration of all the interaction techniques. The participants put on the band and ring devices, and the investigator guided the participants through a repeated process for each technique involving first training, then performing the technique, followed by a short questionnaire. Separating out the techniques in this way allowed us to gain focused feedback from the participants.

After completing this process for all techniques, the mood board canvas was cleared, and participants were asked to author a mood board using the techniques they had just tried (Figure 10). This allowed us to both observe what techniques stuck out to participants, but also provided ecological validity that our techniques support mood board design. Each session lasted 40-70 minutes.

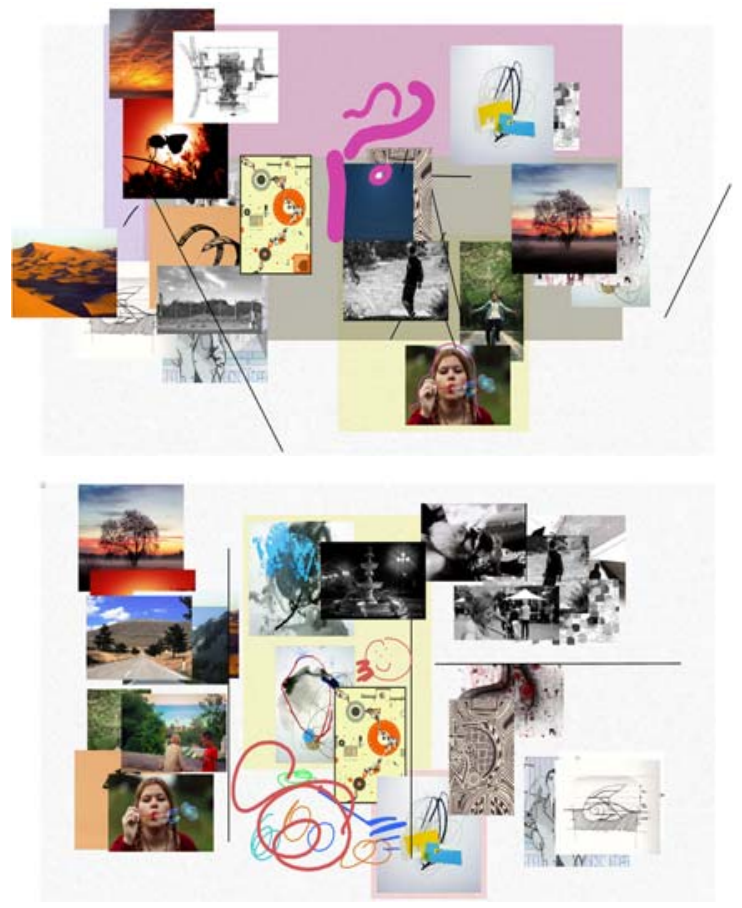

Figure 10. Two mood boards authored by participants.

ChopZ: Most participants found ChopZ easy to use. Many found value in the ability to define a scope and freely arrange the stacking order of elements without having to use some out-of-place widget or repeated contextual menu commands. Some participants reported fatigue issues with maintaining the chop gesture. Many of our bimanual techniques phrase interactions through a sustained left hand gesture. An implication is then that phrasing gestures for longer tasks should have reduced muscle tension. However, this may be particularly challenging on large vertical displays where tension can vary by user depending on user height and where on the display the user interacts.

Straightedge Tool: Participants felt the straightedge tool was quick and easy to use, particularly with the pen. They had no problems understanding the differences between using the pen and using touch. Some participants did express a desire for allowing alignment on the line perpendicular to the straightedge. They wanted the ability to use the straightedge as a virtual wall, preventing elements from moving past the line, as a way of aligning them against the straightedge.

Ink Style Menu: All participants found the in-place ink style menu easy to perform. Some participants did take time getting used to touching with the fingernail. One participant experimented and found the technique was easier for him to perform if he used the middle knuckle (proximal interphalangeal joint) on his index finger [e.g. 31]. 
Single Finger Positioning: Participants were able to quickly pick up on the different positioning operations performed with each hand. They found it intuitive and desirable. During the mood board creation phase at the end of a session, a few participants did report accidently using the wrong hand at first, but felt that with practice, this issue would not occur. This error typically involved using the left hand instead of the right to move a single element. The selection highlight provided quick visual feedback, helping participants recognize that they needed to use the other hand. We suspect that ergonomics - in that one hand is closer to the target element than the other-may influence participants hand choice, particularly with large displays. Prior experiences with touch interfaces where a single touch with either hand moves an element may also be a contributing factor.

Group Sheets: The least preferred technique and the only one that participants reported requiring extra steps to perform was group sheets. Participants felt this technique required more time to learn and understand than the others. Adding too many sheets led to confusion due the visual complexity induced by many overlapping sheets. Only a few participants used group sheets in the final mood board design stage.

Overall Reactions: Participants found our techniques easy to learn and use. The simplest technique, single finger positioning, was almost unanimously the most favored. The techniques involving gestures with different orientations of the hand were hardest for participants to perform, but not remember. We suspect the uniqueness of the gestures made them easier to remember.

\section{DISCUSSION}

\section{Ubiquitous Wearable Devices for Context}

The growing popularity of personal wearables provides emerging avenues for engaging Mark Weiser's ubiquitous computing vision of interconnected wireless devices [61]. Because they exist in a society of interconnected devices, wearables have great potential to shape our interactions with other form-factors, including direct-touch interaction with large displays. It is important to note that a solution based on wearables is truly mobile and personal: wearables move about with users, and remain under users' direct control. Wearables - by their very nature owned by and identified with a specific user - can obviate the identity gap and provide context for lateral preference by distinguishing preferred vs. non-preferred hands.

When a user is uniquely identified, they could walk up to a public display and receive personalized experiences [59]. For example, in an office, a user could simply approach an electronic whiteboard and touch it. We know the user's information from the wearable, and the system could immediately bring up their personal workspace with the last ideas they were working on.

Self-Revelation of Context-Augmented Touch Interaction A key challenge in gestural interfaces is how users learn what interactions are possible and how to perform associated gestures. Through self-revealing gestures [62], we could afford the possible interactions via feedforward techniques (e.g. $[7,23,25])$ that provide revelation of the interactions possible, present dynamic guides within the context of interaction, and require physical rehearsal of the gestures [41]. With wearables, we could identify the user and adjust feedforward and feedback mechanisms accordingly based upon prior experience interacting with a system. We could scaffold learning [29], requiring less revelation and guidance as the user becomes more experienced.

\section{Revisiting Asymmetric vs. Symmetric Interaction}

The main focus of our investigation was on designing bimanual-asymmetric interactions with direct touch that adhere to Guiard's principles. Yet, our goal is not to favor asymmetric over symmetric, but rather to open up a variety of appropriate and consistent mappings. These include both symmetric and asymmetric mappings, as well as the interleaving of the two, as afforded by the additional context our system provides. Affinity and MoodBoard illustrate two contrasting examples of how to achieve this, without ambiguity for bimanual touch input, and without compromising or restricting the interface's design choices.

\section{Flexible Assignment of Roles to the Hands}

While our interactions use strict hand assignments to adhere to Guiard's principles, it is not our intention to argue that strict role assignments are always necessary or even desirable. But armed with additional context for each touch, our approach makes this an intentional design choice rather than an inherent (and oft-unrecognized) ambiguity foisted on the designer by the underlying sensing capabilities (or lack thereof). We seek to empower designers with choices for hand assignments in order to motivate new possibilities and considerations in bimanual interaction design.

\section{ACKNOWLEDGEMENTS}

This material is based upon work supported by the National Science Foundation under grant IIS-1247126. Any opinions, findings, and conclusions expressed in this material are those of the authors and do not reflect the views of the NSF.

\section{CONCLUSION}

We have shown how personal wearables - used to identify hands and users - enable phrasing together direct-touch interactions in a manner consistent with the bimanualasymmetric assignment of roles to the hands as set forth by Guiard. Through our two prototypes, we develop bimanual techniques that demonstrate the interaction possibilities when ambiguity as to who does what (and with which hand) is removed. Our work contributes insights for designing bimanual interactions with lateral preference that has been lacking in standard direct-touch interaction. While we have illustrated several possibilities for augmenting touch with wearables, we hope and anticipate that other possible uses of this input technology may emerge, for touch and beyond.

\section{RIGHTS FOR FIGURES}

Figures 1-10 @ Andrew M. Webb, 2016. 


\section{REFERENCES}

1. Adobe Illustrator. 2016.

http://www.adobe.com/products/illustrator

2. Annett, M., Grossman, T., Wigdor, D. and Fitzmaurice, G. Medusa: a proximity-aware multi-touch tabletop. In Proceedings of the 24th annual ACM symposium on User interface software and technology (2011). ACM, New York, NY, USA, 337-346.

DOI $=10.1145 / 2047196.2047240$

http://doi.acm.org/10.1145/2047196.2047240

3. Apple Watch. 2015. http://www.apple.com/watch

4. Ardito, C., Buono, P., Costabile, M.F. and Desolda, G. Interaction with Large Displays: A Survey. $A C M$ Computing Survey. 47, 3, Article 46 (February 2015), 38 pages. DOI=http://doi.acm.org/10.1145/2682623

5. Azad, A., Ruiz, J., Vogel, D., Hancock, M., and Lank, E. Territoriality and behaviour on and around large vertical publicly-shared displays. In Proceedings of the Designing Interactive Systems Conference (2012).

ACM, New York, NY, USA, 468-477.

DOI $=10.1145 / 2317956.2318025$

http://doi.acm.org/10.1145/2317956.2318025

6. Balakrishnan, R. and Hinckley, K. Symmetric bimanual interaction. In Proceedings of the SIGCHI conference on Human Factors in Computing Systems (2000). ACM, New York, NY, USA, 33-40. DOI=http://dx.doi.org/10.1145/332040.332404

7. Bau, O. and Mackay. W.E. OctoPocus: a dynamic guide for learning gesture-based command sets. In Proceedings of the 21 st annual ACM symposium on User interface software and technology (2008). ACM, New York, NY, USA, 37-46. DOI=http://dx.doi.org/10.1145/1449715.1449724

8. Beyer, H. and Holtzblatt, K. Contextual design.

Interactions 6, 1 (January 1999), 32-42.

DOI $=10.1145 / 291224.291229$

http://doi.acm.org/10.1145/291224.291229

9. Bier, E.A., Stone, M.C., Pier, K., Buxton, W., and DeRose, T.D. Toolglass and magic lenses: the seethrough interface. In Proceedings of the 20th annual conference on Computer graphics and interactive techniques (1993). ACM, New York, NY, USA, 73-80. DOI $=10.1145 / 166117.166126$ http://doi.acm.org/10.1145/166117.166126

10. Blažica, B., Vladušič, D., and Mladenić, D. MTi: A method for user identification for multitouch displays. International Journal of Human-Computer Studies. 71, 6 (June 2013), 691-702.

DOI $=10.1016 /$ j.jihcs.2013.03.002

http://dx.doi.org/10.1016/j.ijhcs.2013.03.002

11. Brandl, P., Forlines, C., Wigdor, D., Haller, M., and Shen, C. Combining and measuring the benefits of bimanual pen and direct-touch interaction on horizontal interfaces. In Proceedings of the working conference on Advanced visual interfaces (2008). ACM, New York, NY, USA, 154-161. DOI=http://dx.doi.org/10.1145/1385569.1385595

12. Buxton, B. Sketching User Experiences: getting the design right and the right design. 2007. Morgan Kaufmann.

13. Buxton, W. Chunking and phrasing and the design of human-computer dialogues. In IFIP Congress, 1986, 475-480.

14. Buxton, W., Fitzmaurice, G., Balakrishnan, R., and Kurtenbach, G. Large Displays in Automotive Design. IEEE Computer Graphics and Applications 20, 4 (July 2000), 68-75. DOI $=10.1109 / 38.851753$ http://dx.doi.org/10.1109/38.851753

15. Buxton, W. and Myers, B. A study in two-handed input. In Proceedings of the SIGCHI Conference on Human Factors in Computing Systems (1986), Marilyn Mantei and Peter Orbeton (Eds.). ACM, New York, NY, USA, 321-326. DOI=10.1145/22627.22390 http://doi.acm.org/10.1145/22627.22390

16. Chatty, S. Extending a graphical toolkit for two-handed interaction. In Proceedings of the 7th annual ACM symposium on User interface software and technology (1994). ACM, New York, NY, USA, 195-204. DOI=http://dx.doi.org/10.1145/192426.192500

17. Chen, X.A., Grossman, T., Wigdor, D.J., and Fitzmaurice, G. Duet: exploring joint interactions on a smart phone and a smart watch. In Proceedings of the SIGCHI Conference on Human Factors in Computing Systems (2014). ACM, New York, NY, USA, 159-168. DOI=http://dx.doi.org/10.1145/2556288.2556955

18. Davidson, P.L. and Han, J.Y. Extending 2D object arrangement with pressure-sensitive layering cues. In Proceedings of the 21 st annual ACM symposium on User interface software and technology (2008). ACM, New York, NY, USA, 87-90.

DOI $=10.1145 / 1449715.1449730$

http://doi.acm.org/10.1145/1449715.1449730

19. Dietz, P. and Leigh, D. DiamondTouch: a multi-user touch technology. In Proceedings of the 14th annual ACM symposium on User interface software and technology (2001). ACM, New York, NY, USA, 219226. DOI $=10.1145 / 502348.502389$

http://doi.acm.org/10.1145/502348.502389

20. Ewerling, P., Kulik, A., and Froehlich, B. Finger and hand detection for multi-touch interfaces based on maximally stable extremal regions. In Proceedings of the 2012 ACM international conference on Interactive tabletops and surfaces (2012). ACM, New York, NY, USA, 173-182.

DOI=http://dx.doi.org/10.1145/2396636.2396663 
21. Fass, A., Forlizzi, J., and Pausch, R. MessyDesk and MessyBoard: two designs inspired by the goal of improving human memory. In Proceedings of the 4th conference on Designing interactive systems: processes, practices, methods, and techniques (2002). ACM, New York, NY, USA, 303-311.

$\mathrm{DOI}=10.1145 / 778712.778754$

http://doi.acm.org/10.1145/778712.778754

22. Fitbit. 2016. https://www.fitbit.com

23. Freeman, D., Benko, H., Morris, M.R., and Wigdor, D. ShadowGuides: visualizations for in-situ learning of multi-touch and whole-hand gestures. In Proceedings of the ACM International Conference on Interactive Tabletops and Surfaces (2009). ACM, New York, NY, USA, 165-172.

DOI=http://dx.doi.org/10.1145/1731903.1731935

24. Garner, S., and McDonagh-Philp, D. Problem Interpretation and Resolution via Visual Stimuli: The Use of 'Mood Boards' in Design Education. Journal of Art \& Design Education, 2001, 20: 57-64. DOI $=10.1111 / 1468-5949.00250$

25. Ghomi, E., Huot, S., Bau, O., Beaudouin-Lafon, M., and Mackay, W.E. Arpège: learning multitouch chord gestures vocabularies. In Proceedings of the 2013 ACM international conference on Interactive tabletops and surfaces (2013). ACM, New York, NY, USA, 209-218. DOI=http://dx.doi.org/10.1145/2512349.2512795

26. Greenberg, S., Marquardt, N., Ballendat, T., DiazMarino, R., and Wang, M. Proxemic interactions: the new ubicomp? interactions 18, 1 (January 2011), 4250. DOI=http://dx.doi.org/10.1145/1897239.1897250

27. Guiard, Y. Asymmetric division of labor in human skilled bimanual action: The kinematic chain as a model. In Journal of motor behavior, 1987, 19(4), 486517.

28. Guimbretière, F., Stone, M., and Winograd, T. Fluid interaction with high-resolution wall-size displays. In Proceedings of the 14th annual ACM symposium on User interface software and technology (2001). ACM, New York, NY, USA, 21-30.

DOI $=10.1145 / 502348.502353$

http://doi.acm.org/10.1145/502348.502353

29. Guzdial, M. Software-realized scaffolding to facilitate programming for science learning. Interactive Learning Environments, 1994, 4(1), 001-044.

30. Hamilton, W., Kerne, A., and Robbins, T. Highperformance pen + touch modality interactions: a realtime strategy game eSports context. In Proceedings of the 25th annual ACM symposium on User interface software and technology (2012). ACM, New York, NY, USA, 309-318. DOI=10.1145/2380116.2380156 http://doi.acm.org/10.1145/2380116.2380156
31. Harrison, C., Schwarz, J., and Hudson, S.E. TapSense: enhancing finger interaction on touch surfaces. In Proceedings of the 24th annual ACM symposium on User interface software and technology (2011). ACM, New York, NY, USA, 627-636.

DOI $=10.1145 / 2047196.2047279$

http://doi.acm.org/10.1145/2047196.2047279

32. Hinckley, K., Pahud, M., Benko, H., Irani, P., Guimbretière, F., Gavriliu, M., Chen, X.A., Matulic, F., Buxton, W., and Wilson, A. 2014. Sensing techniques for tablet+stylus interaction. In Proceedings of the 27th annual ACM symposium on User interface software and technology (2014). ACM, New York, NY, USA, 605-614. DOI=10.1145/2642918.2647379 http://doi.acm.org/10.1145/2642918.2647379

33. Hinckley, K., Yatani, K., Pahud, M., Coddington, N., Rodenhouse, J., Wilson, A., Benko, H., and Buxton, B. 2010. Pen + touch $=$ new tools. In Proceedings of the 23nd annual ACM symposium on User interface software and technology (2010). ACM, New York, NY, USA, 27-36. DOI=10.1145/1866029.1866036 http://doi.acm.org/10.1145/1866029.1866036

34. Hinrichs, U. and Carpendale, S. Gestures in the wild: studying multi-touch gesture sequences on interactive tabletop exhibits. In Proceedings of the SIGCHI Conference on Human Factors in Computing Systems (2011). ACM, New York, NY, USA, 30233032. DOI $=10.1145 / 1978942.1979391$ http://dx.doi.org/10.1145/1978942.1979391

35. Holz, C. and Baudisch, P. Fiberio: a touchscreen that senses fingerprints. InProceedings of the 26th annual ACM symposium on User interface software and technology (2013). ACM, New York, NY, USA, 41-50. DOI=http://dx.doi.org/10.1145/2501988.2502021

36. Ju, W., Lee, B.A., and Klemmer, S.R. Range: exploring implicit interaction through electronic whiteboard design. In Proceedings of the 2008 ACM conference on Computer supported cooperative work (2008). ACM, New York, NY, USA, 17-26. DOI $=10.1145 / 1460563.1460569$ http://doi.acm.org/10.1145/1460563.1460569

37. Kabbash, P., Buxton, W., and Sellen, A. Two-handed input in a compound task. In Proceedings of the SIGCHI Conference on Human Factors in Computing Systems (1994), Beth Adelson, Susan Dumais, and Judith Olson (Eds.). ACM, New York, NY, USA, 417423. DOI $=10.1145 / 191666.191808$ http://doi.acm.org/10.1145/191666.191808

38. Kienzle, W. and Hinckley, K. LightRing: alwaysavailable 2D input on any surface. In Proceedings of the 27th annual ACM symposium on User interface software and technology (2014). ACM, New York, NY, USA, 157-160. DOI=http://dx.doi.org/10.1145/2642918.2647376 
39. Kharrufa, A., Nicholson, J., Dunphy, P., Hodges, S., Briggs, P., and Olivier, P. Using IMUs to Identify Supervisors on Touch Devices. In INTERACT, 2015, Springer, 565-583.

40. Kurtenbach, G., Fitzmaurice, G., Baudel, T., and Buxton, B. The design of a GUI paradigm based on tablets, two-hands, and transparency. In Proceedings of the ACM SIGCHI Conference on Human factors in computing systems (1997). ACM, New York, NY, USA, 35-42. DOI=http://dx.doi.org/10.1145/258549.258574

41. Kurtenbach, G., Moran, T. P., \& Buxton, W. Contextual animation of gestural commands. In Computer Graphics Forum, December 1994, 13(5), Blackwell Science Ltd.

42. Latulipe, C., Mann, S., Kaplan, C.S., and Clarke, C.L.A. symSpline: symmetric two-handed spline manipulation. In Proceedings of the SIGCHI Conference on Human Factors in Computing Systems (2006), Rebecca Grinter, Thomas Rodden, Paul Aoki, Ed Cutrell, Robin Jeffries, and Gary Olson (Eds.). ACM, New York, NY, USA, 349-358. DOI=http://dx.doi.org/10.1145/1124772.1124825

43. Leganchuk, A., Zhai, S., and Buxton, W. Manual and cognitive benefits of two-handed input: an experimental study. ACM Transactions on ComputerHuman Interaction. 5, 4 (December 1998), 326-359. DOI $=10.1145 / 300520.300522$ http://doi.acm.org/10.1145/300520.300522

44. Li, F.C.Y., Guy, R.T., Yatani, K., and Truong, K.N. The 1line keyboard: a QWERTY layout in a single line. In Proceedings of the 24th annual ACM symposium on User interface software and technology (2011). ACM, New York, NY, USA, 461-470. DOI $=10.1145 / 2047196.2047257$ http://doi.acm.org/10.1145/2047196.2047257

45. Lucero, A. Framing, aligning, paradoxing, abstracting, and directing: how design mood boards work. In Proceedings of the Designing Interactive Systems Conference (2012). ACM, New York, NY, USA, 438447. DOI $=10.1145 / 2317956.2318021$ http://doi.acm.org/10.1145/2317956.2318021

46. Lucero, A., Aliakseyeu, D., and Martens, J.B. Funky wall: presenting mood boards using gesture, speech and visuals. In Proceedings of the working conference on Advanced visual interfaces (2008). ACM, New York, NY, USA, 425-428. DOI $=10.1145 / 1385569.1385650$ http://doi.acm.org/10.1145/1385569.1385650

47. Marquardt, N., Kiemer, J., and Greenberg, S. What caused that touch?: expressive interaction with a surface through fiduciary-tagged gloves. In $A C M$ International Conference on Interactive Tabletops and Surfaces (2010). ACM, New York, NY, USA, 139-
142. $\mathrm{DOI}=10.1145 / 1936652.1936680$

http://doi.acm.org/10.1145/1936652.1936680

48. Mare, S., Markham, A.M., Cornelius, C., Peterson, R., and Kotz, D. ZEBRA: zero-effort bilateral recurring authentication. In IEEE Symposium on Security and Privacy (SP), 2014, IEEE 705-720. DOI $=10.1109 /$ SP.2014.51

49. Matulic, F. and Norrie, M.C. Pen and touch gestural environment for document editing on interactive tabletops. In Proceedings of the ACM international conference on Interactive tabletops and surfaces (2013). ACM, New York, NY, USA, 41-50. DOI $=10.1145 / 2512349.2512802$ http://doi.acm.org/10.1145/2512349.2512802

50. Microsoft Band. 2016.

http://www.microsoft.com/microsoft-band

51. Morris, M.R., Huang, A., Paepcke, A., and Winograd, T. Cooperative gestures: multi-user gestural interactions for co-located groupware. In Proceedings of the SIGCHI Conference on Human Factors in Computing Systems (2006), Rebecca Grinter, Thomas Rodden, Paul Aoki, Ed Cutrell, Robin Jeffries, and Gary Olson (Eds.). ACM, New York, NY, USA, 12011210. DOI=http://dx.doi.org/10.1145/1124772.1124952

52. Mynatt, E.D., Igarashi, T., Edwards, W.K., and LaMarca, A. Flatland: new dimensions in office whiteboards. In Proceedings of the SIGCHI conference on Human Factors in Computing Systems (1999). ACM, New York, NY, USA, 346-353. $\mathrm{DOI}=10.1145 / 302979.303108$ http://doi.acm.org/10.1145/302979.303108

53. Ogata, M., Sugiura, Y., Osawa, H., and Imai, M. iRing: intelligent ring using infrared reflection.

In Proceedings of the 25th annual ACM symposium on User interface software and technology (2012). ACM, New York, NY, USA, 131-136.

DOI=http://dx.doi.org/10.1145/2380116.2380135

54. Pedersen, E.R., McCall, K., Moran, T.P., and Halasz, F.G. Tivoli: an electronic whiteboard for informal workgroup meetings. In Proceedings of the Conference on Human Factors in Computing Systems (1993).

ACM, New York, NY, USA, 391-398.

DOI $=10.1145 / 169059.169309$

http://doi.acm.org/10.1145/169059.169309

55. Ramakers, R., Vanacken, D., Luyten, K., Coninx, K., and Schöning, J. Carpus: a non-intrusive user identification technique for interactive surfaces. In Proceedings of the 25th annual ACM symposium on User interface software and technology (2012). ACM, New York, NY, USA, 35-44.

DOI $=10.1145 / 2380116.2380123$

http://doi.acm.org/10.1145/2380116.2380123 
56. Ramos, G., Robertson, G., Czerwinski, M., Tan, D., Baudisch, P., Hinckley, K., and Agrawala, M. Tumble! Splat! helping users access and manipulate occluded content in 2D drawings. In Proceedings of the working conference on Advanced visual interfaces (2006).

ACM, New York, NY, USA, 428-435.

$\mathrm{DOI}=10.1145 / 1133265.1133351$

http://doi.acm.org/10.1145/1133265.1133351

57. Rofouei, M., Wilson, A., Brush, A.J., and Tansley. S. Your phone or mine?: fusing body, touch and device sensing for multi-user device-display interaction. In Proceedings of the SIGCHI Conference on Human Factors in Computing Systems (2012). ACM, New York, NY, USA, 1915-1918. DOI=http://dx.doi.org/10.1145/2207676.2208332

58. Strange, A. Wear this Bluetooth Ring to Control All Your Devices With Your Finger, 2014, http://mashable.com/2014/04/29/nod-bluetooth-ring/

59. Vogel, D. and Balakrishnan, R. Interactive public ambient displays: transitioning from implicit to explicit, public to personal, interaction with multiple users. In Proceedings of the 17th annual ACM symposium on User interface software and technology (2004). ACM, New York, NY, USA, 137-146. DOI $=10.1145 / 1029632.1029656$ http://doi.acm.org/10.1145/1029632.1029656

60. Vogel, D. and Casiez, G. Conté: multimodal input inspired by an artist's crayon. In Proceedings of the 24th annual ACM symposium on User interface software and technology (2011). ACM, New York, NY, USA, 357-366.

DOI=http://dx.doi.org/10.1145/2047196.2047242

61. Weiser, M. The computer for the 21 st century. Scientific American, 1991, 265(3), 94-104.
62. Wigdor, D. and Wixon, D. Design Guidelines: SelfRevealing Multi-Touch Gestures. Brave NUI world: designing natural user interfaces for touch and gesture, 2011, Elsevier: 150-154.

63. Wilkinson, G., Kharrufa, A., Hook, J., Pursglove, B., Wood, G., Haeuser, H., Hammerla, N.Y., Hodges, S., and Olivier, P. Expressy: Using a Wrist-worn Inertial Measurement Unit to Add Expressiveness to Touchbased Interactions. In Proceedings of the $\mathrm{CHI}$ Conference on Human Factors in Computing Systems (2016). ACM, New York, NY, USA, 2832-2844. DOI: http://dx.doi.org/10.1145/2858036.2858223

64. Wu, M., and Balakrishnan, R. Multi-finger and whole hand gestural interaction techniques for multi-user tabletop displays. In Proceedings of the 16th annual ACM symposium on User interface software and technology (2003). ACM, New York, NY, USA, 193202. DOI=10.1145/964696.964718 http://doi.acm.org/10.1145/964696.964718

65. Yang, X.D., Grossman, T., Wigdor, D., and Fitzmaurice, G. Magic finger: always-available input through finger instrumentation. In Proceedings of the 25th annual ACM symposium on User interface software and technology (2012). ACM, New York, NY, USA, 147-156. DOI=10.1145/2380116.2380137 http://doi.acm.org/10.1145/2380116.2380137

66. Zhang, H., Yang, X.D., Ens, B., Liang, H.N., Boulanger, P., and Irani, P. See me, see you: a lightweight method for discriminating user touches on tabletop displays. In Proceedings of the SIGCHI Conference on Human Factors in Computing Systems (2012). ACM, New York, NY, USA, 2327-2336. DOI $=10.1145 / 2207676.2208392$ http://doi.acm.org/10.1145/2207676.2208392 